\title{
DEPTH AND THE LOCAL LANGLANDS CORRESPONDENCE
}

\author{
ANNE-MARIE AUBERT, PAUL BAUM, ROGER PLYMEN, AND MAARTEN SOLLEVELD
}

AbstraCt. Let $G$ be an inner form of a general linear group over a non-archimedean local field. We prove that the local Langlands correspondence for $G$ preserves depths. We also show that the local Langlands correspondence for inner forms of special linear groups preserves the depths of essentially tame Langlands parameters.

\section{Contents}

1. Introduction

2. $\quad$ The local Langlands correspondence for inner forms of $\mathrm{GL}_{n}(F) \quad 3$

2.1. The statement of the correspondence 3

2.2. The Jacquet-Langlands correspondence 5

$\begin{array}{lll}2.3 . & \text { Depth for Langlands parameters } & 7\end{array}$

$\begin{array}{lll}2.4 . & \text { The depth of representations of } \mathrm{GL}_{m}(D) & 8\end{array}$

$\begin{array}{lll}2.5 . & \text { Conductors of representations of } \mathrm{GL}_{m}(D) & 10\end{array}$

$\begin{array}{lll}2.6 . & \text { Depth preservation } & 13\end{array}$

3. The local Langlands correspondence for inner forms of $\mathrm{SL}_{n}(F) \quad 14$

$\begin{array}{lll}3.1 . & \text { The statement of the correspondence } & 14\end{array}$

$\begin{array}{lll}3.2 . & \text { The depth of representations of } \mathrm{GL}_{m}(D)_{\text {der }} & 15\end{array}$

$\begin{array}{lll}3.3 . & \text { The depth of Langlands parameters for } \mathrm{GL}_{m}(D)_{\mathrm{der}} & 16\end{array}$

References

\section{INTRODUCTION}

Let $F$ be a non-archimedean local field and let $G$ be a connected reductive group over $F$. Let $\Phi(G)$ denote the collection of equivalence classes of Langlands parameters for $G$, and $\operatorname{Irr}(G)$ the set of (isomorphism classes of) irreducible smooth $G$-representations. A central role in the representation theory of such groups is played by the local Langlands correspondence (LLC). It is supposed to be a map

$$
\operatorname{Irr}(G) \rightarrow \Phi(G)
$$

that enjoys several naturality properties [Bor, Vog]. The LLC should preserve interesting arithmetic information, like local L-functions and $\epsilon$-factors. A lesser-known invariant that makes sense on both sides of the LLC is depth.

Date: October 5, 2014.

2010 Mathematics Subject Classification. 20G25, 22E50.

Key words and phrases. representation theory, p-adic group, local Langlands program, division algebra.

The second author was partially supported by NSF grant DMS- 1200475 . 
The depth of a Langlands parameter $\phi$ is easy to define. For $r \in \mathbb{R}_{\geq 0}$ let $\operatorname{Gal}\left(F_{s} / F\right)^{r}$ be the $r$-th ramification subgroup of the absolute Galois group of $F$. Then the depth of $\phi$ is the smallest number $d(\phi) \geq 0$ such that $\phi$ is trivial on $\operatorname{Gal}\left(F_{s} / F\right)^{r}$ for all $r>d(\phi)$.

The depth $d(\pi)$ of an irreducible $G$-representation $\pi$ was defined by Moy and Prasad [MoPr1, MoPr2], in terms of filtrations $P_{x, r}\left(r \in \mathbb{R}_{\geq 0}\right)$ of the parahoric subgroups $P_{x} \subset G$. On the basis of several examples (see below) it is reasonable to expect that for many Langlands parameters $\phi \in \Phi(G)$ with L-packet $\Pi_{\phi}(G) \subset \operatorname{Irr}(G)$ one has

$$
d(\phi)=d(\pi) \quad \text { for all } \pi \in \Pi_{\phi}(G)
$$

This relation would be useful for several reasons. Firstly, it allows one to employ some counting arguments in the local Langlands correspondence, because (up to unramified twists) there are only finitely many irreducible representations and Langlands parameters whose depth is at most a specified upper bound.

Secondly, it would be a step towards a more explicit LLC. One can try to determine the groups $P_{x, r} / P_{x, r+\epsilon}(\epsilon>0$ small $)$ and their representations explicitly, and to match them with representations of $\operatorname{Gal}\left(F_{s} / F\right) / \mathrm{Gal}\left(F_{s} / F\right)^{r+\epsilon}$.

Thirdly, one can use (1) as a working hypothesis when trying to establish a local Langlands correspondence, to determine whether or not two irreducible representations stand a chance of belonging to the same L-packet.

The most basic case of depth preservation concerns Langlands parameters $\phi \in$ $\Phi(G)$ that are trivial on both the inertia group $\mathbf{I}_{F}$ and on $\mathrm{SL}_{2}(\mathbb{C})$. These can be regarded as Langlands parameters of negative depth. Such a $\phi$ is only relevant for $G$ if $G$ is quasi-split and splits over an unramified extension of $F$. In that case one can say that an irreducible $G$-representation has negative depth if it possesses a nonzero vector fixed by a hyperspecial maximal compact subgroup. The Satake isomorphism shows how to each such representation one can associate (in a natural way) a Langlands parameter of the above kind.

The $G$-representations of depth zero have been subjected to ample study, see for example GSZ, Mor, DBRe, Mœ. According to Moy-Prasad, an irreducible representation has depth zero if and only if it has nonzero vectors fixed by the prounipotent radical of some parahoric subgroup of $G$. This includes Iwahori-spherical representations and Lusztig's unipotent representations [Lus1, Lus2]. A Langlands parameter has depth zero if and only if it is trivial on the wild inertia subgroup of the absolute Galois group of $F$. For depth zero the equality (1) is conjectured, and proven in certain cases, in [DBRe]. It fits very well with the aforementioned work of Lusztig.

In positive depth there is the result of $\mathrm{Yu}$ [Yu2, $\S 7.10]$, who proved (1) for unramified tori. For $\mathrm{GL}_{n}(F)$, (1) was claimed in [Yu1, §2.3.6] and proved in [ABPS2, Proposition 4.5]. For $\operatorname{GSp}_{4}(F),(1)$ is proved in [Gan, $\left.\S 10\right]$. We refer to [GrRe, Ree, ReYu] for some interesting examples of positive depth Langlands parameters and supercuspidal representations. Most of these examples satisfy (1), but in [ReYu, $\S 7.4-7.5]$ some particular cases are mentioned in which (1) does not hold. All these counterexamples appear in small residual characteristics. So it remains to be seen in which generality the local Langlands correspondence will preserve depths. 
In this paper we will prove that the local Langlands correspondence preserves depth for the inner forms of $\mathrm{GL}_{n}(F)$. In a few non-split cases, this was done before in LaRa]. For inner forms of $\operatorname{SL}_{n}(F)$, we will prove an inequality between depths, which becomes an equality if the Langlands parameter is essentially tame in the sense that it maps the wild inertia group to a maximal torus of $\mathrm{PGL}_{n}(\mathbb{C})$. Every Langlands parameter for an inner form of $\mathrm{SL}_{n}(F)$ is essentially tame if the residual characteristic of $F$ does not divide $n$.

Let $D$ be a division algebra with centre $F$, of dimension $d^{2}$ over $F$. Then $G=$ $\mathrm{GL}_{m}(D)$ is an inner form of $\mathrm{GL}_{n}(F)$ with $n=d m$. There is a reduced norm map Nrd: $\mathrm{GL}_{m}(D) \rightarrow F^{\times}$and the derived group $G_{\mathrm{der}}:=\operatorname{ker}\left(\mathrm{Nrd}: G \rightarrow F^{\times}\right)$is an inner form of $\mathrm{SL}_{n}(F)$. Every inner form of $\mathrm{GL}_{n}(F)$ or $\mathrm{SL}_{n}(F)$ is isomorphic to one of this kind.

The main steps in the proof of our depth-preservation theorem are:

- With the Langlands classification one reduces the problem to essentially square-integrable representations and elliptic Langlands parameters.

- Express the depth in terms of $\epsilon$-factors and conductors. This is a technical step which involves detailed knowledge of the representation theory of $G$. Here it is convenient to use an alternative but equivalent version of depth, the normalized level of an irreducible $G$-representation.

- Show that the Jacquet-Langlands correspondence for $G=\mathrm{GL}_{m}(D)$ preserves $\epsilon$-factors. Since the LLC for $\operatorname{GL}_{m}(D)$ is defined as a composition of the Jacquet-Langlands correspondence with the LLC for $\mathrm{GL}_{n}(F)$ and the latter is known to preserve $\epsilon$-factors, this proves depth-preservation for inner forms of $\mathrm{GL}_{n}(F)$.

- Relate the depth for $G_{\text {der }}$ to depth for $G$. For irreducible representations nothing changes, but for Langlands parameters the depth can decrease if one replaces the dual group $\mathrm{GL}_{n}(\mathbb{C})$ by $\mathrm{PGL}_{n}(\mathbb{C})$. Using several properties of the Artin reciprocity map, we show that such a decrease in depth cannot occur if the Langlands parameter is essentially tame.

This paper develops results presented by the second author in a lecture at the 2013 Arbeitstagung.

Acknowledgements. The authors wish to thank Vincent Sécherre for some helpful explanations on the construction of simple types for $\mathrm{GL}_{m}(D)$, Mark Reeder for pointing out some examples where the depth is not preserved, Wilhelm Zink for explaining properties of the Artin reciprocity map, and Paul Broussous for providing the reference $[\mathrm{BaBr}]$, which has allowed a substantial simplification of the proof of Proposition 2.6 from a previous version.

\section{The local Langlands CORRESPOndence FOR InNer FORMS OF $\operatorname{GL}_{n}(F)$}

\subsection{The statement of the correspondence.}

The local Langlands correspondence for supercuspidal representations of $\mathrm{GL}_{n}(F)$ was established in the important papers [LRS, HaTa, Hen2. Together with the Jacquet-Langlands correspondence this provides the LLC for essentially squareintegrable representations of inner forms $G=\mathrm{GL}_{m}(D)$ of $\mathrm{GL}_{n}(F)$. It is extended to all irreducible $G$-representations via the Zelevinsky classification [Zel, DKV], see [HiSa, ABPS1]. For these groups every L-packet is a singleton and the LLC is a 
canonical bijective map

$$
\operatorname{rec}_{D, m}: \operatorname{Irr}\left(\mathrm{GL}_{m}(D)\right) \rightarrow \Phi\left(\mathrm{GL}_{m}(D)\right)
$$

A remarkable aspect of Langlands' conjectures $\mathrm{Vog}$ is that it is better to consider not just one reductive group at a time, but all inner forms of a given group simultaneously. Inner forms share the same Langlands dual group, so in (2) the right hand side is the same for all inner forms $G$ of the given group. Then one can turn (2) into a bijection by defining a suitable equivalence relation on the set of inner forms and taking the corresponding union of the sets $\operatorname{Irr}(G)$ on the left hand side (see Theorem 2.1 below).

We define the equivalence classes of such inner forms to be in bijection with the isomorphism classes of central simple $F$-algebras of dimension $n^{2}$ via $\mathrm{M}_{m}(D) \mapsto$ $\mathrm{GL}_{m}(D)$, respectively $\mathrm{M}_{m}(D) \mapsto \mathrm{GL}_{m}(D)_{\text {der }}$.

As Langlands dual group of $G$ we take $\mathrm{GL}_{n}(\mathbb{C})$. To deal with inner forms it is advantageous to consider the conjugation action of $\mathrm{SL}_{n}(\mathbb{C})$ on these two groups. It induces a natural action of $\mathrm{SL}_{n}(\mathbb{C})$ on the collection of Langlands parameters for $\mathrm{GL}_{n}(F)$. For any such parameter $\phi$ we can define

$$
C(\phi)=Z_{\mathrm{SL}_{n}(\mathbb{C})}(\operatorname{im} \phi), \text { and } \mathcal{S}_{\phi}=C(\phi) / C(\phi)^{\circ} .
$$

Notice that the centralizers are taken in $\mathrm{SL}_{n}(\mathbb{C})$ and not in the Langlands dual group.

Via the Langlands correspondence the non-trivial irreducible representations of $\mathcal{S}_{\phi}$ are associated to irreducible representations of non-split inner forms of $\mathrm{GL}_{n}(F)$. For example, consider a Langlands parameter $\phi$ for $\mathrm{GL}_{2}(F)$ which is elliptic, that is, whose image is not contained in any torus of $\mathrm{GL}_{2}(\mathbb{C})$. Then $\mathcal{S}_{\phi}=Z\left(\mathrm{SL}_{2}(\mathbb{C})\right) \cong$ $\{ \pm 1\}$. The pair $\left(\phi, \operatorname{triv}_{\mathcal{S}_{\phi}}\right)$ parametrizes an essentially square-integrable representation of $\mathrm{GL}_{2}(F)$ and $\left(\phi, \operatorname{sgn}_{\mathcal{S}_{\phi}}\right)$ parametrizes an irreducible representation of the inner form $D^{\times}$, where $D$ denotes a noncommutative division algebra of dimension 4 over $F$.

The enhanced version of the local Langlands correspondence for all inner forms of general linear groups over nonarchimedean local fields says:

Theorem 2.1. ABPS2, Theorem 1.1]

There is a canonical bijection between:

- pairs $(G, \pi)$ with $\pi \in \operatorname{Irr}(G)$ and $G$ an inner form of $\operatorname{GL}_{n}(F)$, considered up to equivalence;

- $\mathrm{GL}_{n}(\mathbb{C})$-conjugacy classes of pairs $(\phi, \rho)$ with $\phi \in \Phi\left(\mathrm{GL}_{n}(F)\right)$ and $\rho \in$ $\operatorname{Irr}\left(\mathcal{S}_{\phi}\right)$.

Via the Kottwitz isomorphism [Kot, Proposition 6.4] every character of $Z\left(\mathrm{SL}_{n}(\mathbb{C})\right)$ determines a central simple $F$-algebra $\mathrm{M}_{m}(D)$. As $Z\left(\mathrm{SL}_{n}(\mathbb{C})\right) \subset C(\phi)$, for any Langlands parameter as above a character of $\mathcal{S}_{\phi}$ determines an inner form $\mathrm{GL}_{m}(D)$ of $\mathrm{GL}_{n}(F)$. In contrast with the usual LLC, our L-packets for inner forms of general linear groups need not be singletons. To be precise, the packet $\Pi_{\phi}$ contains the unique representation $\operatorname{rec}_{D, m}^{-1}(\phi)$ of $G=\mathrm{GL}_{m}(D)$ if $\phi$ is relevant for $G$, and no $G$ representations otherwise. 


\subsection{The Jacquet-Langlands correspondence.}

A representation $\pi$ of $G$ is called essentially square-integrable if $\left.\pi\right|_{G_{\text {der }}}$ is squareintegrable and there exists an unramified character $\chi$ of $G$ such that $\pi \otimes \chi$ is unitary. We denote the set of (equivalence classes of) irreducible essentially squareintegrable $G$-representations by $\operatorname{Irr}_{\text {ess } L^{2}}(G)$. There is a natural bijection between $\operatorname{Irr}_{\text {ess } L^{2}}\left(\mathrm{GL}_{n}(F)\right)$ and $\operatorname{Irr}_{\text {ess } L^{2}}\left(\mathrm{GL}_{m}(D)\right)$, discovered first for $\mathrm{GL}_{2}(F)$ by Jacquet and Langlands JJaLa. The local Langlands correspondence for $\mathrm{GL}_{m}(D)$ is constructed with the help of the Jacquet-Langlands correspondence. Here we recall some useful properties of the latter correspondence.

Theorem 2.2. Let $\mathrm{GL}_{m}(D)$ be an inner form of $\mathrm{GL}_{n}(F)$. There exists a canonical bijection

$$
\mathrm{JL}: \operatorname{Irr}_{\mathrm{ess} L^{2}}\left(\mathrm{GL}_{n}(F)\right) \rightarrow \operatorname{Irr}_{\mathrm{ess} L^{2}}\left(\mathrm{GL}_{m}(D)\right)
$$

with the following properties:

(a) There is a canonical identification of the semisimple elliptic conjugacy classes in $\mathrm{GL}_{n}(F)$ with those in $\mathrm{GL}_{m}(D)$. Let $g \in \mathrm{GL}_{n}(F)$ and $g^{\prime} \in \mathrm{GL}_{m}(D)$ be semisimple elliptic elements in the same conjugacy class and let $\theta_{\pi}$ be the character of $\pi \in \operatorname{Irr}_{\mathrm{ess} L^{2}}\left(\mathrm{GL}_{n}(F)\right)$. Then

$$
(-1)^{n} \theta_{\pi}(g)=(-1)^{m} \theta_{\mathrm{JL}(\pi)}\left(g^{\prime}\right) .
$$

(b) JL preserves twists with characters of $F^{\times}$:

$$
\mathrm{JL}(\pi \otimes \chi \circ \operatorname{det})=\mathrm{JL}(\pi) \otimes \chi \circ \mathrm{Nrd} .
$$

(c) JL respects contragredients: $\mathrm{JL}\left(\pi^{\vee}\right)=\mathrm{JL}(\pi)^{\vee}$.

(d) Let $P^{\prime}$ be a standard parabolic subgroup of $\mathrm{GL}_{m}(D)$, with Levi factor $M^{\prime}=$ $\prod_{i} \mathrm{GL}_{m_{i}}(D)$. Let $P$ be the corresponding standard parabolic subgroup of $\mathrm{GL}_{n}(F)$, with Levi factor $M=\prod_{i} \mathrm{GL}_{d m_{i}}(F)$. Then the Jacquet modules $r_{P}^{\mathrm{GL}_{n}(F)}(\pi)$ and $r_{P^{\prime}}^{\mathrm{GL}_{m}(D)}(\mathrm{JL}(\pi))$ are either both zero or both irreducible and essentially squareintegrable. In the latter case

$$
\mathrm{JL}\left(r_{P}^{\mathrm{GL}_{n}(F)}(\pi)\right)=r_{P^{\prime}}^{\mathrm{GL}_{m}(D)}(\mathrm{JL}(\pi)) .
$$

In other words, JL and its version for $M$ and $M^{\prime}$ respect Jacquet restriction.

(e) JL preserves supercuspidality.

(f) $\mathrm{JL}\left(\mathrm{St}_{\mathrm{GL}_{n}(F)}\right)=\mathrm{St}_{\mathrm{GL}_{m}(D)}$, where $\mathrm{St}_{G}$ denotes the Steinberg representation of $G$.

$(g)$ JL preserves $\gamma$-factors:

$$
\gamma(s, \operatorname{JL}(\pi), \psi)=\gamma(s, \pi, \psi) \text { for any nontrivial character } \psi \text { of } F .
$$

(h) JL preserves L-functions: $L(s, \mathrm{JL}(\pi))=L(s, \pi)$.

(i) JL preserves $\epsilon$-factors: $\epsilon(s, \mathrm{JL}(\pi), \psi)=\epsilon(s, \pi, \psi)$.

Proof. The correspondence, which is in fact characterized by property (a), is proven over $p$-adic fields in [DKV] and over local fields of positive characteristic in [Bad]. Properties (b) and (c) are obvious in view of (a). The same goes for property (f) in the case $m=1$, because then $\mathrm{St}_{\mathrm{GL}_{m}(D)}$ is just the trivial representation of $D^{\times}$. For (d) see [Bad, §5], in particular Proposition B. Obviously (d) implies (e). Property (f) for $m>1$ follows from (f) for $m=1$ and property (d). Property (g) was proven over local function fields in [Bad, p. 741], with an argument that also works over $p$-adic fields. 
Properties (h) and (i) were claimed in [DKV], with the difference that the $\epsilon$ factors of $\pi$ and $\operatorname{JL}(\pi)$ are said to agree only up to a sign $(-1)^{n+m}$. This sign is due to a convention that does not agree with GoJa], which we use for the definition of $\epsilon$-factors. Unfortunately the argument for (h) and (i) given in [DKV, §B.1] is incorrect. Instead, we will establish (h) by direct calculation.

Let $\nu_{D}$ denote the unramified character $g^{\prime} \mapsto\left\|\operatorname{Nrd} g^{\prime}\right\|_{F}$ of $\mathrm{GL}_{m}(D)$. Consider any $\pi^{\prime} \in \operatorname{Irr}_{\mathrm{ess} L^{2}}\left(\mathrm{GL}_{m}(D)\right)$. By [DKV, §B.2] or [Tad, $\left.\S 2\right]$ there exist:

- integers $a, b, s_{\sigma}$ such that $a b=m$ and $s_{\sigma}$ divides $a d$;

- an irreducible supercuspidal representation $\sigma$ of $\mathrm{GL}_{a}(D)$,

such that $\pi^{\prime}$ is a consituent of the parabolically induced representation

$$
\Pi^{\prime}:=I_{\mathrm{GL}_{a}(D)^{b}}^{\mathrm{GL}_{m}(D)}\left(\nu_{D}^{s_{\sigma} \frac{1-b}{2}} \sigma \otimes \nu_{D}^{s_{\sigma} \frac{3-b}{2}} \sigma \otimes \cdots \otimes \nu_{D}^{s_{\sigma} \frac{b-1}{2}} \sigma\right) .
$$

By [Jac, Proposition 2.3] the L-function of (4) is the product of L-functions of the inducing representations:

$$
L\left(s, \Pi^{\prime}\right)=\prod_{k=1}^{b} L\left(s, \nu_{D}^{s_{\sigma}(k-(1+b) / 2)} \sigma\right)=\prod_{k=1}^{b} L\left(s+s_{\sigma}(k-(1+b) / 2), \sigma\right) .
$$

By definition $L\left(s, \pi^{\prime}\right)^{-1}$ is a monic polynomial in $q^{-s}$, and by [Jac, 2.7.4] it is a factor of the monic polynomial $L\left(s, \Pi^{\prime}\right)^{-1}$. Now there are two cases to be distinguished, depending on whether $\sigma$ is an unramified representation of $D^{\times}$or not.

Case 1: $a=1, b=m$ and $\sigma$ is unramified.

There exists an unramified character $\chi$ of $F^{\times}$such that $\sigma=\chi \circ N r d$. By DKV, $\S \mathrm{B} .2]$ or [Tad, §2] (4) only has an essentially square-integrable subquotient if $s_{\sigma}=d$. Then $\pi^{\prime} \cong \mathrm{St}_{\mathrm{GL}_{m}(D)} \otimes \chi \circ \mathrm{Nrd}$, so $\mathrm{JL}^{-1}\left(\pi^{\prime}\right) \cong S t_{\mathrm{GL}_{n}(F)} \otimes \chi \circ$ det. With property (f) this enables us to compute the $\gamma$-factor. Let $\omega_{F}$ be a uniformizer of $F, \mathfrak{o}_{F}$ the ring of integers and $\mathfrak{p}_{F}$ its maximal ideal. Assume that $\psi$ is trivial on $\mathfrak{p}_{F}$ but not on $\mathfrak{o}_{F}$. Then

$$
\gamma\left(s, \pi^{\prime}, \psi\right)=\gamma\left(s, \operatorname{St}_{\mathrm{GL}_{n}(F)} \otimes \chi \circ \operatorname{det}, \psi\right)=(-1)^{n} q^{n / 2} \frac{1-q^{-s+(1-n) / 2} \chi\left(\omega_{F}\right)}{1-q^{-s+(1+n) / 2} \chi\left(\omega_{F}\right)} .
$$

By [GoJa, Proposition 4.4], (5) becomes

$$
\prod_{k=1}^{m} L(s+d(k-(1+m) / 2), \chi \circ \mathrm{Nrd})=\prod_{k=1}^{m} L(s+d(k-(1+m) / 2)+(d-1) / 2, \chi) .
$$

Now we apply [GoJa, Theorem 7.11.4]. It is stated only for $\mathrm{GL}_{n}(F)$, but the proof with zeros and poles of L-functions goes through because we know $\gamma\left(s, \pi^{\prime}, \psi\right)$. We find that for the L-function of $\pi^{\prime}$ we need only the factor $k=m$ of $(7)$ :

$$
L\left(s, \pi^{\prime}, \psi\right)=L(s+(n-1) / 2, \chi)=\left(1-q^{-s+(1-n) / 2} \chi\left(\omega_{F}\right)\right)^{-1} .
$$

In particular the whole calculation works with $d=1$, so

$$
L\left(s, \mathrm{St}_{\mathrm{GL}_{m}(D)} \otimes \chi \circ \mathrm{Nrd}\right)=L\left(s, \mathrm{St}_{\mathrm{GL}_{n}(F)} \otimes \chi \circ \mathrm{det}\right)=L(s+(n-1) / 2, \chi) .
$$

Case 2: all other $\sigma$.

Then [GoJa, Proposition 5.11] says that $L(s, \sigma \otimes \chi)=1$ for every unramified character $\chi$ of $\mathrm{GL}_{a}(D)$. Hence $L\left(s, \Pi^{\prime}\right)=1$ by (5). We observed above that $L\left(s, \pi^{\prime}\right)^{-1}$ is a factor of $L\left(s, \Pi^{\prime}\right)$, so $L\left(s, \pi^{\prime}\right)=1$. Because $\mathrm{JL}$ is bijective, $\mathrm{JL}^{-1}\left(\pi^{\prime}\right)$ is not an 
unramified twist of the Steinberg representation, so $L\left(s, \mathrm{JL}^{-1}\left(\pi^{\prime}\right)\right)=1$ as well. This proves property $(\mathrm{h})$.

In view of the relation

$$
\epsilon(s, \pi, \psi)=\gamma(s, \pi, \psi) L(s, \pi) L\left(1-s, \pi^{\vee}\right),
$$

(i) follows directy from (c), (g) and (h).

We record a particular consequence of equations (6), (8) and (9):

$$
\epsilon\left(s, \mathrm{St}_{\mathrm{GL}_{m}(D)} \otimes \chi \circ \mathrm{Nrd}, \psi\right)=(-1)^{n-1} \epsilon(s, \chi, \psi)=(-1)^{n-1} q^{s-1 / 2} \chi\left(\omega_{F}^{-1}\right)
$$

for any character $\psi$ of $F$ which is trivial on $\mathfrak{p}_{F}$ but not on $\mathfrak{o}_{F}$.

\subsection{Depth for Langlands parameters.}

Let $F_{s}$ be a separable closure of $F$ and let $\operatorname{Gal}\left(F_{s} / F\right)$ be the absolute Galois group of $F$. We recall some properties of its ramification groups (with respect to the upper numbering), as defined in [Ser, Remark IV.3.1]:

- $\operatorname{Gal}\left(F_{s} / F\right)^{-1}=\operatorname{Gal}\left(F_{s} / F\right)$ and $\operatorname{Gal}\left(F_{s} / F\right)^{0}=\mathbf{I}_{F}$, the inertia group.

- For every $l \in \mathbb{R}_{\geq 0}, \operatorname{Gal}\left(F_{s} / F\right)^{l}$ is a compact subgroup of $\mathbf{I}_{F}$. It consists of all $\gamma \in \operatorname{Gal}\left(F_{s} / \bar{F}\right)$ which, for every finite Galois extension $E$ of $F$ contained in $F_{s}$, act trivially on the ring $\mathfrak{o}_{E} / \mathfrak{p}_{E}^{i(l, E)}$ (where $i(l, E) \in \mathbb{Z}_{\geq 0}$ can be found with [Ser, §IV.3]).

- $l \in \mathbb{R}_{\geq 0}$ is called a jump of the filtration if

$$
\operatorname{Gal}\left(F_{s} / F\right)^{l+}:=\bigcap_{l^{\prime}>l} \operatorname{Gal}\left(F_{s} / F\right)^{l^{\prime}}
$$

does not equal $\operatorname{Gal}\left(F_{s} / F\right)^{l}$. The set of jumps of the filtration is countably infinite and need not consist of integers.

Recall Bor] that a Langlands parameter for $\mathrm{GL}_{m}(D)$ is a continuous homomorphism

$$
\phi: \mathbf{W}_{F} \times \mathrm{SL}_{2}(\mathbb{C}) \rightarrow \mathrm{GL}_{n}(\mathbb{C})
$$

such that:

- $\phi\left(\mathbf{W}_{F}\right)$ consists of semisimple elements;

- $\left.\phi\right|_{\mathrm{SL}_{2}(\mathbb{C})}: \mathrm{SL}_{2}(\mathbb{C}) \rightarrow \mathrm{GL}_{n}(\mathbb{C})$ is a morphism of complex algebraic groups;

- $\phi$ is relevant for $\mathrm{GL}_{m}(D)$. This means that the conjugacy class of a Levi subgroup of $\mathrm{GL}_{n}(\mathbb{C})$ minimally containing $\operatorname{im}(\phi)$ should correspond to a conjugacy of class of Levi subgroups of $\mathrm{GL}_{m}(D)$.

We define the depth of such a Langlands parameter as

$$
d(\phi):=\inf \left\{l \geq 0 \mid \operatorname{Gal}\left(F_{s} / F\right)^{l+} \subset \operatorname{ker} \phi\right\} .
$$

We say that $\phi \in \Phi\left(\mathrm{GL}_{n}(F)\right)$ is elliptic if its image is not contained in any proper Levi subgroup of $\mathrm{GL}_{n}(\mathbb{C})$.

Let $\psi$ be a nontrivial character of $F$ and let $c(\psi)$ be the largest integer $c$ such that $\psi$ is trivial on $\mathfrak{p}_{F}^{-c}$. The $\epsilon$ factor of $\phi($ and $\psi$ ) was defined in [Tat]. It takes the form

$$
\epsilon(s, \phi, \psi)=\epsilon(0, \phi, \psi) q^{-s(a(\phi)+n c(\psi))} \text { with } \epsilon(0, \phi, \psi) \in \mathbb{C}^{\times}
$$


Here $a(\phi) \in \mathbb{Z}_{\geq 0}$ is the Artin conductor of $\phi$ (called $f(\phi)$ in [Ser, §VI.2]). To study $a(\phi)$ it is convenient to rewrite $\phi$ in terms of the Weil-Deligne group. For $\gamma \in \mathbf{W}_{F}$ put

$$
\phi_{0}(\gamma)=\phi(\gamma, 1) \phi\left(1,\left(\begin{array}{cc}
\|\gamma\|^{1 / 2} & 0 \\
0 & \|\gamma\|^{-1 / 2}
\end{array}\right)\right)
$$

so $\phi_{0}$ is a representation of $\mathbf{W}_{F}$ which agrees with $\phi$ on $\mathbf{I}_{F}$. Define $N \in \mathfrak{g l}_{n}(\mathbb{C})$ as the nilpotent element $\log \phi\left(1,\left(\begin{array}{ll}1 & 1 \\ 0 & 1\end{array}\right)\right)$. Then $\left(\phi_{0}, N\right)$ is the Weil-Deligne representation of $\mathbf{W}_{F} \ltimes \mathbb{C}$ corresponding to $\phi$.

Denote the vector space $\mathbb{C}^{n}$ endowed with the representation $\phi$ by $V$, and write $V_{N}=\operatorname{ker}(N: V \rightarrow V)$. By definition [Tat, $\S 4.1 .6$ ]

$$
\begin{aligned}
& a(\phi)=a\left(\phi_{0}\right)+\operatorname{dim}\left(V^{\mathbf{I}_{F}} / V_{N}^{\mathbf{I}_{F}}\right), \\
& \epsilon(s, \phi, \psi)=\epsilon\left(0, \phi_{0}, \psi\right) \operatorname{det}\left(-\left.\operatorname{Frob}\right|_{\left.V^{\mathbf{I}_{F}} / V_{N}^{\mathbf{I}_{F}}\right) q^{-s(a(\phi)+n c(\psi))},}\right.
\end{aligned}
$$

where Frob denotes a geometric Frobenius element of $\mathbf{W}_{F}$.

Lemma 2.3. For any elliptic $\phi \in \Phi\left(G L_{n}(F)\right)$

$$
d(\phi):= \begin{cases}0 & \text { if } \mathbf{I}_{F} \subset \operatorname{ker}(\phi), \\ \frac{a(\phi)}{n}-1 & \text { otherwise, }\end{cases}
$$

Proof. This was proved in ABPS2, Lemma 4.4] under the additional assumption $\mathrm{SL}_{2}(\mathbb{C}) \subset$ ker $\phi$. We will reduce to that special case.

Since $\phi$ is elliptic, it defines an irreducible $n$-dimensional representation $V$ of $\mathbf{W}_{F} \times \mathrm{SL}_{2}(\mathbb{C})$. Hence there are irreducible representations $\left(\phi_{1}, V_{1}\right)$ of $\mathbf{W}_{F}$ and $\left(\phi_{2}, V_{2}\right)$ of $\mathrm{SL}_{2}(\mathbb{C})$ such that

$$
(\phi, V)=\left(\phi_{1}, V_{1}\right) \otimes\left(\phi_{2}, V_{2}\right) .
$$

In particular $V^{\mathbf{I}_{F}}=V_{1}^{\mathbf{I}_{F}} \otimes V_{2}$. Suppose first that $V_{1}^{\mathbf{I}_{F}}=V_{1}$. Then $\mathbf{I}_{F} \subset \operatorname{ker} \phi$, so $d(\phi)=0$ by definition. Now suppose $V_{1}^{\mathbf{I}_{F}} \neq V_{1}$. As $\left(\phi_{1}, V_{1}\right)$ is irreducible and $\mathbf{I}_{F}$ is normal in $\mathbf{W}_{F}$, we must have $V_{1}^{\mathbf{I}_{F}}=0$. Hence $V^{\mathbf{I}_{F}}=0$, which by 12 and (13) implies $a(\phi)=a\left(\phi_{0}\right)$. By [Ser, Corollary VI.2.1'] $a\left(\phi_{0}\right)$ is additive in $V$ and depends only on

$$
\left.\phi_{0}\right|_{\mathbf{I}_{F}}=\left.\phi\right|_{\mathbf{I}_{F}}=\left.\phi_{1}\right|_{\mathbf{I}_{F}} \otimes \mathrm{id}_{V_{2}} .
$$

Now it follows from 16 that

$$
a(\phi)=a\left(\phi_{1}\right) \operatorname{dim} V_{2}=n a\left(\phi_{1}\right) / \operatorname{dim} V_{1} .
$$

As $\operatorname{ker} \phi_{1} \supset \mathrm{SL}_{2}(\mathbb{C})$ we may apply [ABPS2, Lemma 4.4], which together with (17) gives

$$
d\left(\phi_{1}\right)=\frac{a\left(\phi_{1}\right)}{\operatorname{dim} V_{1}}-1=\frac{a(\phi)}{n}-1 .
$$

To conclude, we note that $d\left(\phi_{1}\right)=d(\phi)$ by 16 .

\subsection{The depth of representations of $\mathrm{GL}_{m}(D)$.}

Let $k_{D}=\mathfrak{o}_{D} / \mathfrak{p}_{D}$ be the residual field of $D$. Let $\mathfrak{A}$ be a hereditary $\mathfrak{o}_{F}$-order in $\mathrm{M}_{m}(D)$. The Jacobson radical of $\mathfrak{A}$ will be denoted by $\mathfrak{P}$. Let $r=e_{D}(\mathfrak{A})$ and $e=e_{F}(\mathfrak{A})$ denote the integers defined by $\mathfrak{p}_{D} \mathfrak{A}=\mathfrak{P}^{r}$ and $\mathfrak{p}_{F} \mathfrak{A}=\mathfrak{P}^{e}$, respectively. We have

$$
e_{F}(\mathfrak{A})=d e_{D}(\mathfrak{A})
$$


The normalizer in $G$ of $\mathfrak{A}^{\times}$will be denoted by

$$
\mathfrak{K}(\mathfrak{A}):=\left\{g \in G: g^{-1} \mathfrak{A}^{\times} g=\mathfrak{A}^{\times}\right\} .
$$

Define a sequence of compact open subgroups of $G=\mathrm{GL}_{m}(D)$ by

$$
U^{0}(\mathfrak{A}):=\mathfrak{A}^{\times}, \quad \text { and } \quad U^{j}(\mathfrak{A}):=1+\mathfrak{P}^{j}, \quad j \geq 1 .
$$

Then $\mathfrak{A}^{\times}$is a parahoric subgroup of $G$ and $U^{1}(\mathfrak{A})$ is its pro-unipotent radical. We define the normalized level of an irreducible representation $\pi$ of $G$ to be

$$
d(\pi):=\min \left\{j / e_{F}(\mathfrak{A})\right\},
$$

where $(j, \mathfrak{A})$ ranges over all pairs consisting of an integer $j \geq 0$ and a hereditary $\mathfrak{o}_{F}$-order $\mathfrak{A}$ in $\mathrm{M}_{m}(D)$ such that $\pi$ contains the trivial character of $U^{j+1}(\mathfrak{A})$.

Remark 2.4. When $\pi$ is a representation of $\mathrm{GL}_{n}(F)$, our notion of normalized level coincides with that of [BuHe2, $\S 12.6]$. However when $\pi$ is a representation of $D^{\times}$ (or more generally of $\mathrm{GL}_{m}(D)$ ), the normalized level of $\pi$ as defined above is not equal to the level $\ell_{D}(\pi)$ defined in [BuHe2, $\S 54.1$ ] (resp. $\ell(\pi)$ defined by Broussous in $[\mathrm{BaBr}$, Théorème A.1.2]): we have

$$
d(\pi)=\frac{1}{d} \ell_{D}(\pi) \quad\left(\text { resp. } \quad d(\pi)=\frac{1}{d} \ell(\pi) .\right.
$$

This reflects the fact that we have divided by $e_{F}(\mathfrak{A})$ instead of $e_{D}(\mathfrak{A})$ in Eqn. 19.

The following proposition will allow to use both results that were written in the setting of the normalized level, as general results on the depth in the sense of Moy and Prasad.

Proposition 2.5. The normalized level of $\pi \in \operatorname{Irr}(G)$ equals its Moy-Prasad depth.

Proof. Let us denote the Moy-Prasad depth of $\left(\pi, V_{\pi}\right)$ by $d_{\mathrm{MP}}(\pi)$ for the duration of this proof. For any point $x$ of the Bruhat-Tits building $\mathcal{B}(G)$ of $G$, consider the Moy-Prasad filtrations $P_{x, r}, P_{x, r+}\left(r \in \mathbb{R}_{\geq 0}\right)$ of the parahoric subgroup $P_{x} \subset G$ [MoPr1, §2]. We normalize these filtrations by using the valuation on $F_{s}$ which maps $F^{\times}$onto $\mathbb{Z}$. Then $d_{\mathrm{MP}}(\pi)$ is the minimal $r \in \mathbb{R}_{\geq 0}$ such that $V_{\pi}^{P_{x, r+}} \neq 0$ for some $x \in \mathcal{B}(G)$, see $\operatorname{MoPr2}, \S 3.4]$.

Any hereditary $\mathfrak{o}_{F}$-order $\mathfrak{A}$ in $\mathrm{M}_{m}(D)$ is associated to a unique facet $\mathcal{F}(\mathfrak{A})$ of $\mathcal{B}(G)$. The filtration $\left\{U^{j}(\mathfrak{A}) \mid j \in \mathbb{Z}_{\geq 0}\right\}$ was compared with the Moy-Prasad groups for $x \in \mathcal{F}(\mathfrak{A})$ by Broussous and Lemaire. Let $x_{\mathfrak{A}}$ be the barycenter of $\mathcal{F}(\mathfrak{A})$. From [BrLe, Proposition 4.2 and Appendix A] and the definition of $e_{F}(\mathfrak{A})$ we see that

$$
U^{j}(\mathfrak{A})=P_{x_{\mathfrak{A}}, j / e_{F}(\mathfrak{A})} \text { for all } j \in \mathbb{Z}_{\geq 0} .
$$

Hence the definitions of the normalized level and the Moy-Prasad depth are almost equivalent, the only difference being that for $d_{\mathrm{MP}}(\pi)$ we must consider all points of $\mathcal{B}(G)$, whereas for $d(\pi)$ we may only use barycenters of facets of $\mathcal{B}(G)$. Thus it remains to check the following claim: there exists a facet $\mathcal{F}$ of $\mathcal{B}(G)$ with barycenter $x_{\mathcal{F}}$, such that $V_{\pi}$ has nonzero $P_{x_{\mathcal{F}}, d_{\mathrm{MP}}(\pi)+}$-invariant vectors.

This is easy to see with the explicit constructions of the groups $P_{x, r}$ at hand, but we prefer not to delve into those details here. In fact, since every chamber of $\mathcal{B}(G)$ intersects every $G$-orbit in $\mathcal{B}(G)$, it suffices to consider facets contained in the closure of a fixed "standard" chamber. Then the claim becomes equivalent to saying that $x_{\mathcal{F}}$ is an "optimal point" in the sense of [MoPr1, §6.1]. That is assured by [MoPr1, Remark 6.1], which is applicable because the root system of $G$ is of type $A_{m-1}$. 


\subsection{Conductors of representations of $\mathrm{GL}_{m}(D)$.}

Let $\epsilon(s, \pi, \psi)$ denote the Godement-Jacquet local constant GoJa. It takes the form

$$
\epsilon(s, \pi, \psi)=\epsilon(0, \pi, \psi) q^{-f(\pi, \psi) s}, \quad \text { where } \epsilon(0, \pi, \psi) \in \mathbb{C}^{\times} .
$$

Recall that $c(\psi)$ is the largest integer $c$ such that $\mathfrak{p}_{F}^{-c} \subset \operatorname{ker} \psi$. In the previous section we had $c(\psi)=-1$.

A representation of $D^{\times}$is called unramified if it is trivial on $\mathfrak{o}_{D}^{\times}$. An unramified representation of $D^{\times}$is a character and has depth zero.

Proposition 2.6. Let $\pi$ be a supercuspidal irreducible representation of $G$. We have

$$
f(\pi, \psi)= \begin{cases}n(c(\psi)+1)-1 & \text { if } m=1 \text { and } \pi \text { is unramified, } \\ n(d(\pi)+1+c(\psi)) & \text { otherwise. }\end{cases}
$$

Proof. We suppose first that $m=1$ (so $d=n$ ) and $\pi$ is unramified. The required formula can be read off from (10) if $c(\psi)=-1$. For general $\psi$, applying [BuFr1, Theorem 3.2.11] and taking in account [BuFr1, (1.2.7), (1.2.8), (1.2.10)], we obtain

$$
f(\pi, \psi)=\left(d(1-d-d c(\psi)) \cdot\left(-\frac{1}{d}\right)=d+d c(\psi)-1 .\right.
$$

Hence the first case of Eqn. (21) holds.

From now on, we will assume that $m \geq 2$ or $\pi$ is ramified. Then by combining $\mathrm{BaBr}$, Théorème A.2.1] with the fact that the Godement-Jacquet L-function $L(s, \pi)$ is 1 , we see that $\pi$ satisfies the conditions of Theorem 3.3.8 of [BuFr2]. Recall that $n=m d$. By applying the formula of [BuFr2, Theorem 3.3.8 (iv)], we obtain

$$
q^{f(\pi, \psi)}=\left[\mathfrak{A}: \mathfrak{p}_{F}^{c(\psi)+1} \mathfrak{P}^{j}\right]^{1 / n} .
$$

On the other hand, the $\mathfrak{o}_{F}$-order is $G$-conjugate to the standard principal $\mathfrak{o}_{F}$-order of $\mathrm{M}_{m}(D)$ defined by the partition $(t, \ldots, t)$ ( $r$-times) of $m$, where $m=r t$ and $r=e_{D}(\mathfrak{A})$. Hence we have $\mathfrak{A} / \mathfrak{P} \simeq\left(\mathrm{M}_{t}\left(k_{D}\right)\right)^{r}$. It follows that

$$
[\mathfrak{A}: \mathfrak{P}]=\left(q^{d}\right)^{t^{2} r}=q^{d r t^{2}} .
$$

Hence we get

$$
f(\pi, \psi)=\frac{d r t^{2}(j+e+e c(\psi))}{n}=n\left(\frac{j}{e}+1+c(\psi)\right)
$$

since $d r t^{2}=n t=n^{2} / e$.

On the other hand it follows from [SéSt1, Corollaire 5.22] that there exists a maximal simple type $(J, \lambda)$ in $G$, and an extension $\Lambda$ of $\lambda$ to the normalizer $\bar{J}=$ $\mathrm{N}_{G}(\lambda)$ of $\lambda$, such that

$$
\pi=\mathrm{c}-\operatorname{Ind}_{\bar{J}}^{G} \Lambda
$$

By the construction of the type $(J, \lambda)$, we have $d(\pi) \leq j / e$. Conversely, let $\left[\mathfrak{A}^{\prime}, j^{\prime}, j^{\prime}-\right.$ $\left.1, \beta^{\prime}\right]$ be a stratum contained in $\pi$. Then if $\left[\mathfrak{A}^{\prime}, j^{\prime}, j^{\prime}-1, \beta^{\prime}\right]$ is such that its normalized level $j^{\prime} / e^{\prime}$ is minimal among the normalized levels of all the strata contained in $\pi$, it is necessarily fundamental $[\mathrm{Bro}$, Theorem 1.2.1. (ii)]. Since all the fundamental strata contained in $\pi$ have the same normalized level [BaBr, Théorème A.1.2], we get $j / e=d(\pi)$. 
Theorem 2.7 below proves the validity of Conjecture 4.3 of [LaRa]. In the case when $F$ has characteristic 0 , it is due to Lansky and Raghuram for the groups $\mathrm{GL}_{n}(F)$ and $D^{\times}$, [LaRa, Theorem 3.1], and for certain representations of $\mathrm{GL}_{2}(D)$, LaRa, Theorem 4.1]. Our proof is inspired by those of these results.

Theorem 2.7. The depth $d(\pi)$ and the conductor $f(\pi):=f(\pi, \psi)-n c(\psi)$ of each essentially square-integrable irreducible representation $\pi$ of $\mathrm{GL}_{m}(D)$ are linked by the following relation:

$$
d(\pi)= \begin{cases}0 & \text { if } \pi \text { is an unramified twist of } \operatorname{St}_{\mathrm{GL}_{m}(D)}, \\ \frac{f(\pi)-n}{n} & \text { otherwise. }\end{cases}
$$

In particular

$$
d(\pi)=\max \left\{\frac{f(\pi)-n}{n}, 0\right\} .
$$

Proof. Let $\pi \in \operatorname{Irr}_{\text {ess } L^{2}}\left(\mathrm{GL}_{m}(D)\right)$. We use the same notation as for $\pi^{\prime}$ in the proof of Theorem 2.2.h, so $\pi$ is consituent of

$$
I_{\mathrm{GL}_{a}(D)^{b}}^{\mathrm{GL}}\left(\nu_{D}^{s_{\sigma} \frac{(1-b)}{2}} \sigma \otimes \nu_{D}^{s_{\sigma} \frac{(3-b)}{2}} \sigma \otimes \cdots \otimes \nu_{D}^{s_{\sigma} \frac{(b-1)}{2}} \sigma\right),
$$

where $\sigma \in \operatorname{Irr}\left(\mathrm{GL}_{a}(D)\right)$ is supercuspidal. Since the depth is preserved by parabolic induction [MoPr2, Theorem 5.2], we get

$$
d(\pi)=d\left(\nu_{D}^{s_{\sigma} \frac{(1-b)}{2}} \sigma \otimes \nu_{D}^{s_{\sigma} \frac{(3-b)}{2}} \sigma \otimes \cdots \otimes \nu_{D}^{s_{\sigma} \frac{(b-1)}{2}} \sigma\right) .
$$

It follows that

$$
d(\pi)=d(\sigma) .
$$

We will apply Proposition 2.6 to the supercuspidal representation $\sigma$ of $\mathrm{GL}_{a}(D)$. In the special case $\sigma$ is an unramified representation of $D^{\times}$(hence $a=1$ in this case), Eqn. 21) gives

$$
f(\sigma, \psi)=d(c(\psi)+1)-1,
$$

that is, $f(\sigma)=d-1$. Hence we get

$$
\frac{f(\sigma)-d}{d}=-\frac{1}{d} .
$$

Then it implies that

$$
\max \left\{\frac{f(\sigma)-d}{d}, 0\right\}=\max \left\{-\frac{1}{d}, 0\right\}=0=d(\sigma),
$$

in other words, Eqn. (23) holds for the unramified representations of $D^{\times}$.

In the other cases (that is, $a \neq 1$ or $\sigma$ is ramified), 21) gives $f(\sigma)=a d(d(\sigma)+1)$, that is,

$$
\frac{f(\sigma)}{a d}=d(\sigma)+1 .
$$

Since $d(\sigma) \geq 0$ (by definition of the depth), we obtain that

$$
d(\sigma)=\max \left\{\frac{f(\sigma)-a d}{a d}, 0\right\} .
$$

Hence (22) holds for every supercuspidal irreducible representation of $\mathrm{GL}_{a}(D)$, with $a \geq 1$ an arbitrary integer. 
Recall that $s_{\sigma}$ is an integer dividing $a d$, say $a d=a^{*} s_{\sigma}$ with $a^{*} \in \mathbb{Z}$. The image $\mathrm{JL}^{-1}(\sigma)$ of $\sigma$ under the Jacquet-Langlands correspondence is equivalent to the Langlands quotient of the parabolically induced representation

$$
I_{\mathrm{GL}_{a^{*}(F)^{s}}}^{\mathrm{GL}_{a^{*} s_{\sigma}}(F)}\left(\nu_{F}^{\frac{\left(1-s_{\sigma}\right)}{2}} \sigma^{*} \otimes \nu_{F}^{\frac{\left(3-s_{\sigma}\right)}{2}} \sigma^{*} \otimes \cdots \otimes \nu_{F}^{\frac{\left(s_{\sigma}-1\right)}{2}} \sigma^{*}\right),
$$

where $\sigma^{*}$ is a unitary supercuspidal irreducible representation of $\mathrm{GL}_{a^{*}}(F)$ and $\nu_{F}\left(g^{*}\right)=\left|\operatorname{det}\left(g^{*}\right)\right|_{F}$.

The representation $\mathrm{JL}^{-1}(\pi)$ is equivalent to a constituent of the parabolically induced representation

$$
I_{\mathrm{GL}_{a^{*}}(F)^{\mathrm{GL}_{\sigma}}}^{\mathrm{GL}_{a d b}(F)}\left(\nu_{F}^{\frac{\left(1-b s_{\sigma}\right)}{2}} \sigma^{*} \otimes \nu_{F}^{\frac{\left(3-b s_{\sigma}\right)}{2}} \sigma^{*} \otimes \cdots \otimes \nu_{F}^{\frac{\left(b s_{\sigma}-1\right)}{2}} \sigma^{*}\right) .
$$

We recall from $[\mathrm{Hen}, \S 2.6]$ the formula describing the epsilon factor of $\mathrm{JL}^{-1}(\pi)$ in terms of the local factors of $\sigma^{*}$ :

$$
\epsilon\left(s, \mathrm{JL}^{-1}(\pi), \psi\right)=\prod_{i=0}^{b s_{\sigma}-1} \epsilon\left(s+i, \sigma^{*}, \psi\right) \prod_{j=0}^{b s_{\sigma}-2} \frac{L\left(-s-j, \tilde{\sigma}^{*}\right)}{L\left(s+j, \sigma^{*}\right)} .
$$

Since the Jacquet-Langlands correspondence preserves the $\epsilon$-factors (see Theorem 2.2 i) we have

$$
\epsilon\left(s, \mathrm{JL}^{-1}(\pi), \psi\right)=\epsilon(s, \pi, \psi) .
$$

Thus we have obtained the following formula

$$
\epsilon(s, \pi, \psi)=\prod_{i=0}^{b s_{\sigma}-1} \epsilon\left(s+i, \sigma^{*}, \psi\right) \prod_{j=0}^{b s_{\sigma}-2} \frac{L\left(-s-j, \tilde{\sigma}^{*}\right)}{L\left(s+j, \sigma^{*}\right)} .
$$

If $\pi=\mathrm{St}_{\mathrm{GL}_{m}(D)} \otimes \chi$ for some unramified character $\chi$ of $D^{\times}$, it follows from 10 that $f(\pi, \psi)=-1$ in the case where $c(\psi)=-1$, hence we obtain

$$
f(\pi)=n-1 .
$$

From now on we assume $\pi$ is not equivalent to a representation of the form $\operatorname{St}_{\mathrm{GL}_{m}(D)} \otimes$ $\chi$, with $\chi$ an unramified character of $D^{\times}$(that is, we have $m \neq 1$ or $\sigma$ ramified). Then Theorem 2.2 $\mathrm{b}$ and $\mathrm{f}$ implies that similarly $\mathrm{JL}^{-1}(\pi)$ is not a twist of $\mathrm{St}_{\mathrm{GL}_{n}(F)}$ by an unramified character of $F^{\times}$. Thus we have $a^{*} \neq 1$ or $\sigma^{*}$ ramified. It follows that $L\left(-s-j, \tilde{\sigma}^{*}\right)=L\left(s+j, \sigma^{*}\right)=1$, and we obtain from 28 that

$$
f(\pi)=b s_{\sigma} f\left(\sigma^{*}\right) .
$$

In the special case when $b=1$ the equation 30 gives

$$
f(\sigma)=s_{\sigma} f\left(\sigma^{*}\right) .
$$

Then using (24) and (26) we get

$$
d(\pi)=d(\sigma)=\max \left\{\frac{b s_{\sigma} f\left(\sigma^{*}\right)-b a d}{b a d}, 0\right\}=\max \left\{\frac{f(\pi)-n}{n}, 0\right\} .
$$




\subsection{Depth preservation.}

Corollary 2.8. The Jacquet-Langlands correspondence preserves the depth of essentially square-integrable representations of $\mathrm{GL}_{m}(D)$.

Proof. Theorem 2.2 i shows in particular that the Jacquet-Langlands correspondence preserves conductors. Now Theorem 2.7 shows that it preserves depths as well.

Theorems 2.7 and 2.2 are also the crucial steps to show that the local Langlands correspondence for inner forms of $\mathrm{GL}_{m}(D)$ preserves depths. With similar considerations we show that it also preserves L-functions, $\epsilon$-factors and $\gamma$-factors. We abbreviate these three to "local factors". For the basic properties of the local factors of Langlands parameters we refer to [Tat].

Theorem 2.9. The local Langlands correspondence for representations of $\mathrm{GL}_{m}(D)$ preserves L-functions, $\epsilon$-factors, $\gamma$-factors and depths. In other words, for every irreducible smooth representation $\pi$ of $\mathrm{GL}_{m}(D)$ :

$$
\begin{aligned}
& L(s, \pi)=L\left(s, \operatorname{rec}_{D, m}(\pi)\right), \\
& \epsilon(s, \pi, \psi)=\epsilon\left(s, \operatorname{rec}_{D, m}(\pi), \psi\right), \\
& \gamma(s, \pi, \psi)=\gamma\left(s, \operatorname{rec}_{D, m}(\pi), \psi\right) \\
& d(\pi)=d\left(\operatorname{rec}_{D, m}(\pi)\right) .
\end{aligned}
$$

Proof. It is well-known that the local Langlands correspondence for $\mathrm{GL}_{n}(F)$ preserves local factors, see the introduction of [HaTa].

Assume first that $\pi$ is essentially square-integrable. Recall the notations of the $\epsilon$ factors of $\pi$ and of $\phi:=\operatorname{rec}_{D, m}(\pi) \in \Phi\left(\mathrm{GL}_{m}(D)\right)$ from (11) and (20). By definition

$$
\operatorname{rec}_{D, m}(\pi)=\operatorname{rec}_{F, n}\left(\mathrm{JL}^{-1}(\pi)\right),
$$

so by Theorem $2.2 \operatorname{rec}_{D, m}$ preserves the $\epsilon$-factors of $\pi$ :

$$
\epsilon(0, \phi, \psi) q^{-s(a(\phi)+n c(\psi))}=\epsilon(s, \phi, \psi)=\epsilon(s, \pi, \psi)=\epsilon(0, \pi, \psi) q^{-s f(\pi, \psi)} .
$$

Hence, with the notation from Theorem 2.7

$$
f(\pi)=f(\pi, \psi)-n c(\psi)=a(\phi) .
$$

The properties of $\operatorname{rec}_{F, n}$ imply that $\phi$ is elliptic. By combining Lemma 2.3 with Theorem 2.7 and (34), we obtain that $d(\phi)=d(\pi)$ whenever $\pi$ is essentially squareintegrable.

Now let $\pi$ be any irreducible representation of $\mathrm{GL}_{m}(D)$. By the Langlands classification, there exist a parabolic subgroup $P \subset \mathrm{GL}_{m}(D)$ with Levi factor $M$ and an irreducible essentially square-integrable representation $\omega$ of $M$, such that $\pi$ is a quotient of $I_{P}^{\mathrm{GL}_{m}(D)}(\omega)$. Moy and Prasad proved in [MoPr2, Theorem 5.2] that $\pi$ and $\omega$ have the same depth. By [Jac, Theorem 3.4] $\pi$ and $\omega$ have the same L-functions and $\epsilon$-factors and by [Jac, (2.3) and (2.7.3)] they also have the same $\gamma$-factors.

On the other hand, $M$ is isomorphic to a product of groups of the form $\operatorname{GL}_{m_{i}}(D)$, so the local Langlands correspondence for $M$ is simply the product of that for the $\operatorname{GL}_{m_{i}}(D)$. The Langlands parameters $\operatorname{rec}_{D, m}(\pi)$ and $\operatorname{rec}_{M}(\omega)$ are related via an inclusion of the complex dual groups $\prod_{i} \mathrm{GL}_{d m_{i}}(\mathbb{C}) \rightarrow \mathrm{GL}_{n}(\mathbb{C})$. Hence these two Langlands parameters also have the same depth and local factors. 
Because we already proved that the LLC preserves depths for essentially squareintegrable representations of $\mathrm{GL}_{m}(D)$ or $M$, we can conclude that

$$
d(\pi)=d(\omega)=d\left(\operatorname{rec}_{M}(\omega)\right)=d\left(\operatorname{rec}_{D, m}(\pi)\right),
$$

and similarly for the local factors.

\section{The local Langlands CORRESPOndence For inner forms of $\mathrm{SL}_{n}(F)$}

\subsection{The statement of the correspondence.}

Recall that $F$ is a non-archimedean local field and that the equivalence classes of inner forms of $\mathrm{SL}_{n}(F)$ are in bijection with the isomorphism classes of central simple $F$-algebras of dimension $n^{2}$, via $\mathrm{M}_{m}(D) \mapsto \mathrm{GL}_{m}(D)_{\text {der }}$. As mentioned after Theorem 2.1, every character of $Z\left(\mathrm{SL}_{n}(\mathbb{C})\right)$ gives rise to such an algebra via the Kottwitz isomorphism.

The local Langlands correspondence for $\mathrm{GL}_{m}(D)_{\text {der }}$ is implied by that for $\mathrm{GL}_{m}(D)$, in the following way. A Langlands parameter

$$
\phi: \mathbf{W}_{F} \times \mathrm{SL}_{2}(\mathbb{C}) \rightarrow \mathrm{PGL}_{n}(\mathbb{C})
$$

which is relevant for $\mathrm{GL}_{m}(D)_{\text {der }}$ can be lifted it to a Langlands parameter

$$
\bar{\phi}: \mathbf{W}_{F} \times \mathrm{SL}_{2}(\mathbb{C}) \rightarrow \mathrm{GL}_{n}(\mathbb{C})
$$

which is relevant for $\operatorname{GL}_{m}(D)$, by Wei]. Then $\operatorname{rec}_{m, D}^{-1}(\bar{\phi})$ is an irreducible representation of $\mathrm{GL}_{m}(D)$ which, upon restriction to $\mathrm{GL}_{m}(D)_{\text {der }}$, decomposes as a finite direct sum of irreducible representations. The packet $\Pi_{\phi}\left(\mathrm{GL}_{m}(D)_{\text {der }}\right)$ is defined as the set of irreducible constituents of $\operatorname{Res}_{\mathrm{GL}_{m}(D)}^{\mathrm{GL}_{m}(D)} \operatorname{rec}_{m, D}^{-1}(\bar{\phi})$.

For these groups it is more interesting to consider the enhanced Langlands correspondence, where $\phi$ is supplemented with an irreducible representation of a finite group. In addition to the groups defined in $(3)$, we write

$$
\mathcal{Z}_{\phi}=Z\left(\mathrm{SL}_{n}(\mathbb{C})\right) / Z\left(\mathrm{SL}_{n}(\mathbb{C})\right) \cap C(\phi)^{\circ} \cong Z\left(\mathrm{SL}_{n}(\mathbb{C})\right) C(\phi)^{\circ} / C(\phi)^{\circ} .
$$

Any character of $\mathcal{Z}_{\phi}$ determines a character of $Z\left(\mathrm{SL}_{n}(\mathbb{C})\right)$, and hence an inner form of $\mathrm{SL}_{n}(F)$. An enhanced Langlands parameter is a pair $(\phi, \rho)$ with $\rho \in \operatorname{Irr}\left(\mathcal{S}_{\phi}\right)$. The groups in (3), (35) are related to the more usual component group

$$
S_{\phi}:=Z_{\mathrm{PGL}_{n}(\mathbb{C})}(\operatorname{im} \phi) / Z_{\mathrm{PGL}_{n}(\mathbb{C})}(\operatorname{im} \phi)^{\circ}
$$

by the short exact sequence

$$
1 \rightarrow \mathcal{Z}_{\phi} \rightarrow \mathcal{S}_{\phi} \rightarrow S_{\phi} \rightarrow 1
$$

Hence $\mathcal{S}_{\phi}$ has more irreducible representations than $S_{\phi}$. Via the enhanced Langlands correspondence the additional ones are associated to irreducible representations of non-split inner forms of $\mathrm{SL}_{n}(F)$. The following result is due to Hiraga and Saito [HiSa, Theorem 12.7] for generic representations of $\mathrm{GL}_{m}(D)$ when char $F=0$.

Theorem 3.1. ABPS2, Theorem 1.2]

There exists a bijective correspondence between:

- pairs $\left(\mathrm{GL}_{m}(D)_{\mathrm{der}}, \pi\right)$ with $\pi \in \operatorname{Irr}\left(\mathrm{GL}_{m}(D)_{\mathrm{der}}\right)$ and $\mathrm{GL}_{m}(D)_{\mathrm{der}}$ an inner form of $\mathrm{SL}_{n}(F)$, considered up to equivalence;

- $\mathrm{SL}_{n}(\mathbb{C})$-conjugacy classes of pairs $(\phi, \rho)$ with $\phi \in \Phi\left(\mathrm{SL}_{n}(F)\right)$ and $\rho \in \operatorname{Irr}\left(\mathcal{S}_{\phi}\right)$. 
Here the group $\mathrm{GL}_{m}(D)_{\text {der }}$ determines $\left.\rho\right|_{\mathcal{Z}_{\phi}}$ and conversely. The correspondence satisfies the desired properties from [Bor, $\$ 10.3]$, with respect to restriction from inner forms of $\mathrm{GL}_{n}(F)$, temperedness and essential square-integrability of representations.

We remark that the above bijection need not be canonical if $\Pi_{\phi}\left(\mathrm{GL}_{m}(D)_{\text {der }}\right)$ has more than one element.

\subsection{The depth of representations of $\mathrm{GL}_{m}(D)_{\mathrm{der}}$.}

For the depth of an irreducible representation of $\mathrm{GL}_{m}(D)$ der there are two candidates. Besides the Moy-Prasad depth one can define the normalized level, just as in (19). This was done for representations of $\mathrm{SL}_{n}(F)$ in $\mathrm{BuKu}$. However, Proposition 2.5 quickly reveals that these two notions agree:

Proposition 3.2. The Moy-Prasad depth of an irreducible representation of $\mathrm{GL}_{m}(D)_{\text {der }}$ equals its normalized level.

Proof. Let us compare the descriptions of the two kinds of depth with those given in the proof of Proposition 2.5. By definition $\mathrm{GL}_{m}(D)$ and $\mathrm{GL}_{m}(D)$ der have the same Bruhat-Tits building. The Moy-Prasad depth is defined in terms of the groups

$$
P_{x, r}^{\prime}=P_{x, r} \cap \mathrm{GL}_{m}(D)_{\text {der }} \quad \text { with } \quad x \in \mathcal{B}\left(G L_{m}(D)\right) .
$$

The normalized level is expressed with the groups

$$
U^{j}(\mathfrak{A})^{\prime}=U^{j}(\mathfrak{A}) \cap \mathrm{GL}_{m}(D)_{\text {der }},
$$

where $\mathfrak{A}$ is a hereditary $\mathfrak{o}_{F}$-order in $M_{m}(D)$. With these groups instead of $P_{x, r}$ and $U^{j}(\mathfrak{A})$ the entire proof of Proposition 2.5 carries over to $\mathrm{GL}_{m}(D)_{\text {der }}$.

It turns out that the depth of an irreducible $\operatorname{GL}_{m}(D)_{\text {der-representation }} \pi$ behaves nicely with respect to restriction from $\mathrm{GL}_{m}(D)$. To be precise, equals the minimum of the depths of the irreducible $\mathrm{GL}_{m}(D)$-representations that contain $\pi$. (Notice that this minimum is always attained because all depths for inner forms of $\mathrm{GL}_{n}(F)$ lie in $\frac{1}{n} \mathbb{Z}$.)

Proposition 3.3. Let $\pi \in \operatorname{Irr}\left(\operatorname{GL}_{m}(D)_{\text {der }}\right)$ and let $\bar{\pi} \in \operatorname{Irr}\left(\mathrm{GL}_{m}(D)\right)$ be such that

- $\pi$ is a direct summand of $\operatorname{Res}_{\left.\mathrm{GL}_{m}(D)\right)_{\mathrm{der}}}^{\mathrm{GL}_{m}(D)}(\bar{\pi})$;

- $d(\bar{\pi}) \leq d(\bar{\pi} \otimes \chi \circ \mathrm{Nrd})$ for every character $\chi$ of $F^{\times}$.

Then $d(\pi)=d(\bar{\pi})$.

Proof. In the case $G=\mathrm{GL}_{n}(F)$, this is guaranteed by Proposition 3.2 and $\mathrm{BuKu}$, Proposition 1.7.iii]. The same proof works for $\mathrm{GL}_{m}(D)$ but this would be cumbersome, one would have to check that everything in [BuKu, p.265-268] also works with a division algebra instead of a field.

Instead, we select some parts of [BuKu, $\S 1]$ to provide a shorter proof. Pick a $x \in \mathcal{B}(G)$ such that $(\bar{\pi}, V)$ has nonzero vector fixed by $P_{x, d(\bar{\pi})+}$. Then

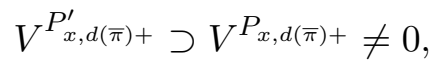

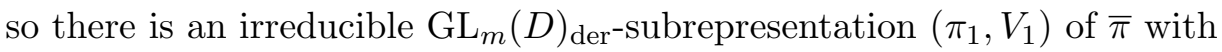

$$
V_{1}^{P_{x, d(\bar{\pi})+}^{\prime}} \neq 0 \quad \text { and } \quad d\left(\pi_{1}\right) \leq d(\bar{\pi}) .
$$


Since $\bar{\pi}$ is irreducible, $\pi_{1}$ is isomorphic to a $\mathrm{GL}_{m}(D)$-conjugate of $\pi$. Conjugation by $g \in \mathrm{GL}_{m}(D)$ sends any Moy-Prasad group $P_{y, r}$ to $P_{g(y), r}$. So this operation preserves depths and

$$
d(\pi)=d\left(\pi_{1}\right) \leq d(\bar{\pi}) .
$$

Suppose now that $d(\pi)<d(\bar{\pi})$. Take a nonzero $v \in V^{P_{x, d(\pi)+}^{\prime}}$ and consider

$$
V_{v}:=\operatorname{span}\left\{\bar{\pi}(g) v \mid g \in P_{x, d(\pi)+}\right\} .
$$

As $P_{x, d(\pi)+}^{\prime}$ is normal in $P_{x, d(\pi)+}$, it acts trivially on $V_{v}$, and $V_{v}$ can be regarded as representation of

$$
P_{x, d(\pi)+} / P_{x, d(\pi)+}^{\prime} \cong \operatorname{Nrd}\left(P_{x, d(\pi)+}\right) \subset F^{\times} .
$$

Hence there is a character $\chi$ of $F^{\times}$such that $\chi^{-1} \circ$ Nrd appears in the action of $P_{x, d(\pi)+}$ on $V_{v}$. Then the irreducible $\mathrm{GL}_{m}(D)$-representation $\bar{\pi} \otimes \chi \circ \operatorname{Nrd}$ has a nonzero vector fixed by $P_{x, d(\pi)+}$, so

$$
d(\bar{\pi} \otimes \chi \circ \mathrm{Nrd}) \leq d(\pi)<d(\bar{\pi}) .
$$

This contradicts the assumptions of proposition, so (37) must be an equality.

\subsection{The depth of Langlands parameters for $\mathrm{GL}_{m}(D)_{\mathrm{der}}$.}

The depth of a Langlands parameter $\phi: \mathbf{W}_{F} \times \mathrm{SL}_{2}(\mathbb{C}) \rightarrow \mathrm{PGL}_{n}(\mathbb{C})$ for an inner form of $\mathrm{SL}_{n}(F)$ is defined as in Section 2.3 .

$$
d(\phi)=\inf \left\{l \in \mathbb{R}_{\geq 0} \mid \operatorname{Gal}\left(F_{s} / F\right)^{l+} \subset \operatorname{ker} \phi\right\} .
$$

The following result may be considered as the non-archimedean analogue of [ChKa, Theorem 1] in the case of the geometric local Langlands correspondence.

Corollary 3.4. Let $\pi \in \operatorname{Irr}\left(\mathrm{GL}_{m}(D)_{\text {der }}\right)$ with Langlands parameter $\phi \in \Phi\left(\mathrm{SL}_{n}(F)\right)$. Then $d(\pi) \geq d(\phi)$.

Proof. Let $\bar{\pi}$ be as in Proposition 3.3 , so $d(\bar{\pi})=d(\pi)$. Put $\bar{\phi}=\operatorname{rec}_{D, m}(\bar{\pi})$, this is a lift of $\phi$ to $\mathrm{GL}_{n}(\mathbb{C})$ and Theorem 2.9 says that $d(\bar{\phi})=d(\bar{\pi})$.

We remark that by the compatibility of the LLC with character twists

$$
d(\bar{\phi}) \leq d(\bar{\phi} \otimes \gamma) \text { for every character } \gamma \text { of } \mathbf{W}_{F} .
$$

For any lift $\bar{\phi} \in \Phi\left(\operatorname{GL}_{n}(F)\right)$ of $\phi$ we have $\operatorname{ker} \bar{\phi} \subset \operatorname{ker} \phi$, so $d(\bar{\phi}) \geq d(\phi)$.

It is possible that the inequality in Corollary 3.4 is strict. The following example was pointed out to the authors by Mark Reeder.

Example 3.5. Take $F=\mathbb{Q}_{2}$ and a Langlands parameter $\phi: \mathbf{W}_{\mathbb{Q}_{2}} \rightarrow \mathrm{PGL}_{2}(\mathbb{C})$ which is trivial on $\mathrm{SL}_{2}(\mathbb{C})$ and has image isomorphic to the symmetric group $S_{4}$. (Such a L-parameter exists, see for example Wei.) We claim that $d(\phi)=1 / 3$.

Let Ad denote the adjoint representation of $\mathrm{PGL}_{2}(\mathbb{C})$ on $\mathfrak{s l}_{2}(\mathbb{C})=\operatorname{Lie}\left(\mathrm{PGL}_{2}(\mathbb{C})\right)$. Then Ado $\phi$ is an irreducible 3-dimensional representation of $\mathbf{W}_{\mathbb{Q}_{2}}$. Since $\mathrm{PGL}_{2}(\mathbb{C})$ is the adjoint group of $\mathfrak{s l}_{2}(\mathbb{C})$, Ado $\phi$ has the same kernel and hence the same depth as $\phi$. One can check that $\operatorname{Ad}\left(\phi\left(\mathbf{I}_{F}\right)\right) \cong A_{4}$ and that the image of the wild inertia subgroup $\mathbf{P}_{F}$ is isomorphic to the Klein four group. With the formula GrRe, (1)] for the Artin conductor we find that $a(\operatorname{Ad} \circ \phi)=4$. By Lemma 2.3 (with $n=3$ ) $d(\operatorname{Ad} \circ \phi)=1 / 3$. 
Let $\bar{\phi}: \mathbf{W}_{\mathbb{Q}_{2}} \rightarrow \mathrm{GL}_{2}(\mathbb{C})$ be a lift of $\phi$. This is an irreducible 2-dimensional representation. We claim that $d(\bar{\phi}) \geq 1 / 2$.

With a suitable basis transformation we can achieve that

$$
\phi\left(\mathbf{P}_{\mathbb{Q}_{2}}\right)=\left\{\left(\begin{array}{ll}
1 & 0 \\
0 & 1
\end{array}\right),\left(\begin{array}{cc}
0 & i \\
i & 0
\end{array}\right),\left(\begin{array}{cc}
-i & 0 \\
0 & i
\end{array}\right),\left(\begin{array}{cc}
0 & 1 \\
-1 & 0
\end{array}\right)\right\} \subset \mathrm{PGL}_{2}(\mathbb{C}) .
$$

Let $1, w_{2}, w_{3}, w_{4} \in \mathbf{P}_{\mathbb{Q}_{2}}$ be preimages of these elements. Irrespective of the choice of the lift of $\phi$ we have

$$
\left[\bar{\phi}\left(w_{3}\right), \bar{\phi}\left(w_{4}\right)\right]=\left[\left(\begin{array}{cc}
-i & 0 \\
0 & i
\end{array}\right),\left(\begin{array}{cc}
0 & 1 \\
-1 & 0
\end{array}\right)\right]=\left(\begin{array}{cc}
-1 & 0 \\
0 & -1
\end{array}\right) \in \mathrm{GL}_{2}(\mathbb{C}) .
$$

Put $E=F_{s}^{\text {ker } \bar{\phi}}$ and endow $\operatorname{Gal}\left(E / \mathbb{Q}_{2}\right) \cong \bar{\phi}\left(\mathbf{W}_{\mathbb{Q}_{2}}\right)$ with the lower numbered filtration. The image of $\mathbf{P}_{\mathbb{Q}_{2}}$ is $\operatorname{Gal}\left(E / \mathbb{Q}_{2}\right)_{1}$ and $\left[p_{3}, p_{4}\right] \in\left[\operatorname{Gal}\left(E / \mathbb{Q}_{2}\right)_{1}, \operatorname{Gal}\left(E / \mathbb{Q}_{2}\right)_{1}\right]$. By Ser, Propostion IV.2.10] $\left[p_{3}, p_{4}\right] \in \operatorname{Gal}\left(E / \mathbb{Q}_{2}\right)_{3}$, so $\bar{\phi}$ is nontrivial on this ramification group. If we lift $\phi$ with as little ramification as possible, $\bar{\phi}\left(\mathbf{W}_{\mathbb{Q}_{2}}\right)$ is an index 2 central extension of $S_{4}$. Writing $d_{j}=\left|\bar{\phi}\left(\operatorname{Gal}\left(E / \mathbb{Q}_{2}\right)_{j}\right)\right|$, we have

$$
d_{0}=24, d_{1}=8, d_{2}=d_{3}=2 \text { and } d_{j}=1 \text { for } j>3 .
$$

The formula GrRe, (1)] gives

$$
a(\bar{\phi})=\frac{\operatorname{dim}(\bar{\phi})}{d_{0}} \sum_{j \geq 0: d_{j}>1} d_{j}=\frac{2}{24}(24+8+2+2)=3 .
$$

Now Lemma 2.3 says that $d(\bar{\phi})=1 / 2$.

To show that Corollary 3.4 is in many cases an equality, we will make use of several well-known properties of the Artin reciprocity map $\mathbf{a}_{F}: \mathbf{W}_{F} \rightarrow F^{\times}$. In particular:

Theorem 3.6. $\mathbf{a}_{F}\left(\operatorname{Gal}\left(F_{s} / F\right)^{l}\right)=U_{F}^{\lceil l\rceil}$ for all $l \in \mathbb{R}_{\geq 0}$.

Proof. For any finite abelian extension $E / F$, [Ser, Corollary 3 to Theorem XV.2.1] says that the Artin reciprocity map gives an isomorphism

$$
\mathbf{a}_{F}: \operatorname{Gal}(E / F)^{l} \rightarrow U_{F}^{\lceil l\rceil} /\left(\mathrm{N}_{E / F}\left(E^{\times}\right) \cap U_{F}^{\lceil l\rceil}\right) .
$$

Let $F_{s}^{\mathrm{ab}}$ be the maximal abelian extension of $F$ contained in $F_{s}$. Taking the projective limit of (39) over all finite, Galois subextensions of $F_{s}^{\mathrm{ab}} / F$, we obtain an isomorphism

$$
\mathbf{a}_{F}: \operatorname{Gal}\left(F_{s}^{\mathrm{ab}} / F\right)^{l} \rightarrow U_{F}^{\lceil l\rceil} .
$$

We note that $\operatorname{Gal}\left(F_{s}^{\mathrm{ab}} / F\right)$ is the quotient of $\operatorname{Gal}\left(F_{s} / F\right)$ modulo the closure of its commutator subgroup. Hence $\mathbf{a}_{F}: \mathbf{W}_{F} \rightarrow F^{\times}$factors via $\operatorname{Gal}\left(F_{s}^{\mathrm{ab}} / F\right)$.

Recall from [BuHe1] that a Langlands parameter for $\mathrm{GL}_{n}(F)$ is essentially tame if its restriction to the wild inertia subgroup $\mathbf{P}_{F}$ of $\mathbf{W}_{F}$ is a direct sum of characters. Clearly $\bar{\phi}$ is essentially tame if and only if $\bar{\phi}\left(\mathbf{P}_{F}\right)$ lies in a maximal torus of $\mathrm{GL}_{n}(\mathbb{C})$, which in turn is equivalent to $\phi\left(\mathbf{P}_{F}\right)$ lying in a maximal torus of $\mathrm{PGL}_{n}(\mathbb{C})$.

Definition 3.7. A Langlands parameter $\phi$ for an inner form of $\mathrm{SL}_{n}(F)$ is essentially tame if $\phi\left(\mathbf{P}_{F}\right)$ lies in a maximal torus of $\mathrm{PGL}_{n}(\mathbb{C})$.

By [BuHe1, Corollary A.4] any L-parameter for (an inner form of) $\mathrm{GL}_{n}(F)$ is essentially tame if the residual characteristoc of $F$ does not divide $n$. Our definition is such that the same holds for Langlands parameters for (inner forms of) $\mathrm{SL}_{n}(F)$.

For such L-parameters the LLC does preserve depths: 
Theorem 3.8. Let $\phi \in \Phi\left(\mathrm{SL}_{n}(F)\right)$ be essentially tame and relevant for $\mathrm{GL}_{m}(D)$ der . Then $d(\pi)=d(\phi)$ for every $\pi \in \Pi_{\phi}\left(\mathrm{GL}_{m}(D)_{\mathrm{der}}\right)$.

Proof. Let $\bar{\phi}$ be as in $(38)$, so $d(\bar{\phi})=d(\pi)$.

First we consider the case where $\bar{\phi}$ is an irreducible $n$-dimensional representation of $\mathbf{W}_{F}$. By [BuHe1, Theorem A.3] there exist a finite, tamely ramified Galois extension $E / F$ and a smooth character $\xi: \mathbf{W}_{E} \rightarrow \mathbb{C}^{\times}$such that $\bar{\phi}=\operatorname{ind}_{\mathbf{W}_{E}} \mathbf{W}_{F}$. We may and will assume that $E$ is contained in our chosen separable closure $F_{s}$ of $F$. By Mackey's induction-restriction formula

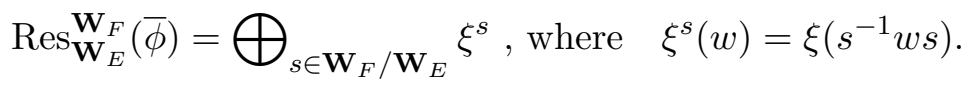

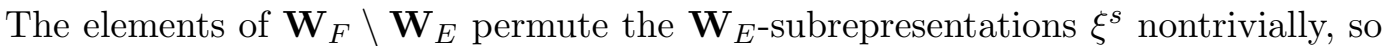
they cannot lie in the kernel of $\bar{\phi}$ :

$$
\operatorname{ker} \bar{\phi}=\left\{w \in \mathbf{W}_{E}: \xi^{s}(w)=1 \forall s \in \mathbf{W}_{F}\right\} .
$$

Let pr: $\mathrm{GL}_{n}(\mathbb{C}) \rightarrow \mathrm{PGL}_{n}(\mathbb{C})$ be the canonical projection. Then $\phi=\operatorname{pr} \circ \bar{\phi}$ and

$$
\operatorname{ker} \phi=\bar{\phi}^{-1}\left(Z\left(\mathrm{GL}_{n}(\mathbb{C})\right)\right)=\left\{w \in \mathbf{W}_{E}: \xi^{s}(w)=\xi(w) \forall s \in \mathbf{W}_{F}\right\} .
$$

Suppose that $d(\bar{\phi})>d(\phi)$. In view of the definition of $d(\phi)$,

$$
\operatorname{ker} \bar{\phi} \supset \mathbf{W}_{E} \cap \operatorname{Gal}\left(F_{s} / F\right)^{d(\bar{\phi})+} \text {, but } \operatorname{ker} \bar{\phi} \not \supset \mathbf{W}_{E} \cap \operatorname{Gal}\left(F_{s} / F\right)^{d(\bar{\phi})} \subset \operatorname{ker} \phi .
$$

The relation between the upper and the lower numbering of the filtration subgroups of $\mathbf{W}_{F}$ [Ser, §IV.3], combined with the compatibility of the lower numbering with subgroups [Ser, Proposition IV.1.2], provides a $l \in \mathbb{R}_{\geq 0}$ such that

$$
\mathbf{W}_{E} \cap \operatorname{Gal}\left(F_{s} / F\right)^{d(\bar{\phi})}=\operatorname{Gal}\left(F_{s} / E\right)^{l} .
$$

In fact $l>0$ because $d(\bar{\phi})>d(\phi) \geq 0$. Since $\operatorname{Res}_{\mathbf{W}_{E}}^{\mathbf{W}_{F}} \bar{\phi}$ is a direct sum of characters, it factors through the Artin reciprocity map $\mathbf{a}_{E}: \mathbf{W}_{E} \rightarrow E^{\times}$. With (41) we see that

$$
\mathbf{a}_{E}\left(\operatorname{Gal}\left(F_{s} / E\right)^{l}\right) \neq \mathbf{a}_{E}\left(\operatorname{Gal}\left(F_{s} / E\right)^{l+}\right) .
$$

By Theorem 3.6 applied to $F_{s} / E, l$ must be a positive integer. When we transfer the conjugation action of $\mathbf{W}_{F}$ on $\mathbf{W}_{E}$ to $E^{\times}$via Artin reciprocity, it becomes the standard action of $\operatorname{Gal}(E / F) \cong \mathbf{W}_{F} / \mathbf{W}_{E}$ on $E^{\times}$. Now says that $\xi$ is a $\operatorname{Gal}(E / F)$-invariant character of $U_{E}^{l}$. Since $l \in \mathbb{Z}_{>0}$ and $E / F$ is tamely ramified, $U_{E}^{l}$ is a cohomologically trivial $\mathrm{Gal}(E / F)$-module. According to BuHe1, Lemma A.1], these properties imply that $\xi$ factors through the norm map $\mathrm{N}_{E / F}$, and there is a unique smooth character

$$
\xi^{\prime} \text { of } U_{E}^{l} \cap F^{\times}=\mathrm{N}_{E / F}\left(U_{E}^{l}\right) \text { such that } \xi=\xi^{\prime} \circ \mathrm{N}_{E / F} \text { on } U_{E}^{l} .
$$

Since $F^{\times} / \operatorname{ker}\left(\xi^{\prime}\right)$ is a finitely generated abelian group and $\mathbb{C}^{\times}$is divisible, we can extend $\xi^{\prime}$ to a smooth character of $F^{\times}$. Via Artin reciprocity this yields a character $\xi_{F}$ of $\mathbf{W}_{F}$. From (42) and the commutative diagram [Ser, $\S$ XI.3]

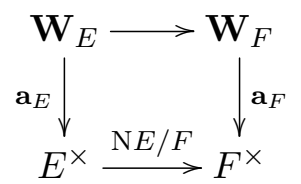


we see that $\xi_{F}=\xi$ on $\operatorname{Gal}\left(F_{s} / E\right)^{d(\bar{\phi})} \cap \mathbf{W}_{E}$. Then $\bar{\phi} \otimes \xi_{F}^{-1}$ is another lift of $\phi$ to $\Phi\left(\mathrm{GL}_{n}(F)\right)$, and $\operatorname{ker} \bar{\phi} \otimes \xi_{F}^{-1}$ contains $\operatorname{Gal}\left(F_{s} / E\right)^{d(\bar{\phi})}$. Thus $d\left(\bar{\phi} \otimes \xi_{F}^{-1}\right)<d(\bar{\phi})$, which contradicts the definition of $\bar{\phi}$. We have shown that $d(\bar{\phi})=d(\phi)$ if $\left.\bar{\phi}\right|_{\mathbf{W}_{F}}$ is irreducible.

For a general essentially tame parameter $\bar{\phi}$ for $\mathrm{GL}_{n}(F),\left.\bar{\phi}\right|_{\mathbf{W}_{F}}$ is a direct sum of irreducible essentially tame parameters $\psi_{i}$ for $\mathrm{GL}_{n_{i}}(F)$, with $n_{i} \leq n$. Writing $\psi_{i}=\operatorname{ind}_{\mathbf{W}_{E_{i}}}^{\mathbf{W}_{F}} \xi_{i}$ as above, we obtain from that

$$
\operatorname{ker} \phi=\left\{w \in \cap_{i} \mathbf{W}_{E_{i}}: \xi_{i}^{s}(w)=\xi_{j}(w) \text { for all } i, j \text { and all } s \in \mathbf{W}_{F}\right\} .
$$

In general this is smaller than

$$
\operatorname{ker}\left(\oplus_{i} \operatorname{pr}_{i} \circ \psi_{i}\right)=\left\{w \in \cap_{i} \mathbf{W}_{E_{i}}: \xi_{i}^{s}(w)=\xi_{i}(w) \text { for all } i \text { and all } s \in \mathbf{W}_{F}\right\},
$$

where $\operatorname{pr}_{i}: \mathrm{GL}_{n_{i}}(\mathbb{C}) \rightarrow \mathrm{PGL}_{n_{i}}(\mathbb{C})$ denotes the canonical projection. Comparing all these kernels we deduce that

$$
\max _{i} d\left(\psi_{i}\right)=d(\bar{\phi}) \geq d(\phi) \geq d\left(\oplus_{i} \operatorname{pr}_{i} \circ \psi_{i}\right)=\max _{i} d\left(\operatorname{pr}_{i} \circ \psi_{i}\right)
$$

However, we cannot just twist $\bar{\phi}$ with a character of $\mathbf{W}_{F}$ derived from the most ramified of the $\psi_{i}$ as in the irreducible case, because that could make the depth of another $\psi_{j}$ much larger.

We suppose once again that $d(\bar{\phi})>d(\phi)$. Then

$$
\operatorname{ker} \bar{\phi} \supset \operatorname{Gal}\left(F_{s} / F\right)^{d(\bar{\phi})+} \text {, but } \operatorname{ker} \bar{\phi} \not \supset \operatorname{Gal}\left(F_{s} / F\right)^{d(\bar{\phi})} \subset \operatorname{ker} \phi .
$$

By (43) all the $\xi_{i}$ agree on $\operatorname{Gal}\left(F_{s} / F\right)^{d(\bar{\phi})}$. The above method produces characters $\xi_{i}^{\prime}$ of $U_{E_{i}}^{d_{i}} \cap F^{\times}$, which agree on $\mathbf{a}_{F}\left(\mathbf{W}_{F}^{d(\bar{\phi})}\right)$. Put $\xi^{\prime}=\left.\xi_{i}^{\prime}\right|_{\mathbf{a}_{F}\left(\mathbf{W}_{F}^{d(\bar{\phi})}\right)}$. Now the same argument as in the irreducible case leads to a contradiction with (38).

\section{REFERENCES}

[ABPS1] A.-M. Aubert, P. Baum, R.J. Plymen, M. Solleveld, "Geometric structure and the local Langlands conjecture", arXiv:1211.0180.

[ABPS2] A.-M. Aubert, P.F. Baum, R.J. Plymen, M. Solleveld, "The local Langlands correspondence for inner forms of $\mathrm{SL}_{n} "$, preprint, arXiv:1305.2638, 2013.

[Bad] A. I. Badulescu, "Correspondance de Jacquet-Langlands pour les corps locaux de caractéristique non nulle", Ann. Sci. Éc. Norm. Sup. (4) 35 (2002), 695-747.

$[\mathrm{BaBr}]$ A. I. Badulescu, with an appendix by P. Broussous, "Un théorème de finitude", Compositio Math. 132 (2002) 177-190.

[Bor] A. Borel, "Automorphic L-functions", Proc. Symp. Pure Math 33.2 (1979), 27-61.

[Bro] P. Broussous, "Minimal strata for GL $\mathrm{GL}_{m}(D)$ ", J. reine angew. Math. 514 (1999), 199-236.

[BrLe] P. Broussous, B. Lemaire, "Building of $\mathrm{GL}(m, D)$ and centralizers", Transformations Groups 7, No. 1 (2002), 15-50.

[BuFr1] C.J. Bushnell, A. Frölich, "Gauss sums and p-adic division algebras", Lecture Notes in Math. 987, Springer-Verlag, 1983.

[BuFr2] C.J. Bushnell, A. Frölich, "Non-abelian congruence Gauss sums and p-adic simple algebras", Proc. London Math. Soc. (3) 50 (1985), 207-264.

[BuHe1] C.J. Bushnell, G. Henniart, "The essentially tame local Langlands correspondence, I", J. Amer. Math. Soc. 18.3 (2005), 685-710.

[BuHe2] C.J. Bushnell, G. Henniart, The local Langlands conjecture for GL(2), Grundlehren der mathematischen Wissenschaften, 335, Springer-Verlag, 2006.

[BuKu] C.J. Bushnell, P.C. Kutzko, "The admissible dual of SL(N) I". Ann. scient. Éc. Norm. Sup. (4) 26 (1993), 261-280 
[ChKa] T.H. Chen, M. Kamgarpour, "Preservation of depth in local geometric Langlands correspondence", arXiv: 1404.0598.

[DBRe] S. DeBacker, M. Reeder, "Depth zero supercuspidal L-packets and their stability", Ann. of Math. 169.3 (2009), 795-901.

[DKV] P. Deligne, D. Kazhdan, M.-F. Vigneras, "Représentations des algèbres centrales simples p-adiques", pp. 33-117 in: Représentations des groupes réductifs sur un corps local, Travaux en cours, Hermann, 1984.

[Gan] R. Ganapathy, "The local Langlands correspondence for $G S p_{4}$ over local function fields", preprint, arXiv:1305.6088, 2013.

[GoJa] R. Godement, H. Jacquet, Zeta functions of simple algebras, Lecture Notes in Mathematics 260, Springer-Verlag, 1972.

[GSZ] M. Grabitz, A.J. Silberger, W. Zink, "Level zero types and Hecke algebras for local central simple algebras", J. Number Theory 91 (2001), 92-125.

[GrRe] B.H. Gross, M. Reeder, "Arithmetic invariants of discrete Langlands parameters", Duke Math. J. 154 (2010), 431-508.

[HaTa] M. Harris, R. Taylor, "The geometry and cohomology of some simple Shimura varieties", Annals of Math. Studies 151, Princeton University Press, Princeton NJ, 2001.

[Hen] G. Henniart, "On the local Langlands conjecture for GL $(n)$ : The cyclic case", Ann. of Math. 123 (1986), 143-203.

[Hen2] G. Henniart, "Une preuve simple de conjectures de Langlands pour GL $(n)$ sur un corps p-adique", Inv. Math. 139 (2000), 439-455.

[HiSa] K. Hiraga, H. Saito, "On L-packets for inner forms of SL ${ }_{n}$ ", Mem. Amer. Math. Soc. 1013, Vol. 215 (2012).

[Jac] H. Jacquet, "Principal L-functions of the linear group", Proc. Symp. Pure Math 33.2 (1979), 63-86.

[JaLa] H. Jacquet, R. Langlands, Automorphic forms on GL(2), Lecture Notes in Mathematics 114, Springer-Verlag, 1970

[Kot] R.E. Kottwitz, "Stable trace formula: cuspidal tempered terms", Duke Math. J. 51.3 (1984), $611-650$.

[LaRa] J. Lansky, A. Raghuram, "On the correspondence of representations between GL $(n)$ and division algebras", Proc. Amer. Math. Soc. 131.5 (2002), 1641-1648.

[LRS] G. Laumon, M. Rapoport, U. Stuhler, "D-elliptic sheaves and the Langlands correspondence", Invent. Math. 113 (1993), 217-238.

[Lus1] G. Lusztig, "Classification of unipotent representations of simple p-adic groups", Int. Math. Res. Notices 11 (1995), 517-589.

[Lus2] G. Lusztig, "Classification of unipotent representations of simple p-adic groups II", Represent. Theory 6 (2002), 243-289.

[Mœ] C. Mœglin, "Stabilité en niveau 0, pour les groupes orthogonaux impairs p-adiques", Documenta Math. 9 (2004), 527-564.

[Mor] L. Morris, "Tamely ramified intertwining algebras", Invent. Math. 114.1 (1993), 1-54.

[MoPr1] A. Moy, G. Prasad, "Unrefined minimal K-types for p-adic groups", Inv. Math. 116 (1994), 393-408.

[MoPr2] A. Moy, G. Prasad, "Jacquet functors and unrefined minimal K-types", Comment. Math. Helvetici 71 (1996), 98-121.

[Ree] M. Reeder, "Supercuspidal L-packets of positive depth and twisted Coxeter elements", J. reine angew. Math. 620 (2008), 1-33.

[ReYu] M. Reeder, J.-K. Yu, "Epipelagic representations and invariant theory", J. Amer. Math. Soc. 27 (2014), 437-477.

[SéSt1] V. Sécherre, S. Stevens, "Représentations lisses de $\mathrm{GL}_{m}(D)$ IV: représentations supercuspidales", J. Inst. Math. Jussieu 7.3 (2008), 527-574.

[Ser] J.-P. Serre, Corps locaux. Hermann, Paris, 1962.

[Tad] M. Tadić, "Induced representations of $\operatorname{GL}(n, A)$ for $p$-adic division algebras $A$ ", J. reine angew. Math. 405 (1990), 48-77.

[Tat] J. Tate, "Number theoretic background", Proc. Symp. Pure Math 33.2 (1979), 3-26.

[Vog] D. Vogan, "The local Langlands conjecture", pp. 305-379 in: Representation theory of groups and algebras, Contemp. Math. 145, American Mathematical Society, Providence RI, 1993. 
[Wei] A. Weil, "Exercices dyadiques", Inv. Math. 27 (1974), 1-22.

[Yu1] J.-K. Yu, "Bruhat-Tits theory and buildings", pp. 53-77 in: Ottawa lectures on admissible representations of reductive p-adic groups, Fields Institute Monographs, American Mathemical Society, 2009

[Yu2] J.-K. Yu, "On the local Langlands correspondence for tori", pp. 177-183 in: Ottawa lectures on admissible representations of reductice p-adic groups, Fields Institute Monographs, American Mathemical Society, 2009

[Zel] A.V. Zelevinsky, "Induced representations of reductive $p$-adic groups II. On irreducible representations of GL(n)", Ann. Sci. École Norm. Sup. (4) 13.2 (1980), 165-210.

Institut de Mathématiques de Jussieu - Paris Rive Gauche, U.M.R. 7586 Du C.N.R.S., U.P.M.C., 4 PLACE Jussieu 75005 PARIS, FranCE

E-mail address: anne-marie.aubert@imj-prg.fr

Mathematics Department, Pennsylvania State University, University Park, PA 16802, USA

E-mail address: baum@math.psu.edu

School of Mathematics, Southampton University, Southampton SO17 1BJ, England and School of Mathematics, Manchester University, Manchester M13 9PL, England

E-mail address: r.j.plymen@soton.ac.uk plymen@manchester.ac.uk

Radboud Universiteit Nijmegen, HeyendaAlseweg 135, 6525AJ Nijmegen, the NetherLANDS

E-mail address: m.solleveld@science.ru.nl 